\title{
Processing and evaluation of measured data from Build-Up systems with special regard to various designs
}

\author{
Michael Wagner ${ }^{1}$, Falk Tegtmeier $^{2}$ \\ 1 Physikalisch-Technische Bundesanstalt, Bundesallee 100, 38116 Braunschweig \\ michael.wagner@ptb.de \\ 2 Physikalisch-Technische Bundesanstalt, Bundesallee 100, 38116 Braunschweig
}

\begin{abstract}
AMA_Conferences_Headline"):
The largest force standard in Europe with a nominal force of $16.5 \mathrm{MN}$ and an uncertainty of $1 \times 10^{-4}$ is located in PTB, Braunschweig. Since there is a high demand for calibration in the meganewton range, this force scale needs to be exceeded. To realize this, the use of large bu-systems as transfer standards is considered [1]. A reliable uncertainty budget is a basic requirement for the traceability of force in the meganewton range using bu-systems. In order to create such an uncertainty budget, a method for the evaluation of measured data is necessary. An important aspect to be considered in this context is the broad variety of design principles of bu-systems.
\end{abstract}

Key words: bu-systems, transfer standards, uncertainty budget, traceability of force, data processing

\section{Designs of BU-Systems}

A force transducer bu-system is a parallel interconnection of force transducers in order to measure a broad range of force using one single type of transducer. For the introduction of the force into the system a wide variety of adaptation parts is being used. All these different shapes or layouts of bu-systems provide a very particular behavior regarding e.g. repeatability, span and hysteresis due to the kinematic behavior of the entire system.

Up to now there is only little knowledge about the influence of the particular design of the parts of bu-systems on the measurement results. These influences will be investigated within the EMRPProject "SIB63 - Force traceability within the meganewton range"

There are six basic components, which are considered to define the design of a bu-system. These are as follows:

- Transducer (number, layout, nominal force)

- Plates for interconnection

- Centering

- Decoupling of parasitic forces

- Decoupling of redundant forces

- Device for averaging the measuring value

The plates for interconnection are the adaptation parts which connect the single transducers to one system. These are usually shaped as plane plates on top and bottom of the system. Beyond this there are systems using three plates with additional decoupling options in between. The centering is a part of the plates that hold the single transducers in their unique position. A decoupling of parasitic forces can be realized by using pendulum or single spherical caps ontop of the transducers. A decoupling of redundant forces is advisable if there are more than three transducers which leads to a static over determination of the system. Differences in the design of these components lead to different behavior of the entire system.

\section{Agreement of a Linguistic usage}

There are two standards, for 'calibrating force transducers' (DIN EN ISO376) and 'verification of static uniaxial testing machines' (DIN EN ISO 7500-1). The metrological examination of large scale build-up systems represents a particular application which is not clearly covered by these both standards. Firstly BU-systems should be used as transfer standards for the traceability of testing-machines, which is the main idea of using them. In this case the evaluation following ISO7500-1 takes into consideration and the force which is indicated by the BU-system then applies as the 'correct force' $F$.

The largest force standard at PTB is $16.5 \mathrm{MN}$, beyond that a traceable calibration of $\mathrm{BU}$ systems is not possible in an uncertainty range under $0.1 \%$. A standardized procedure for extrapolation of BU-system-measuring values 
does not exist yet. For the mentioned reasons, BU-systems are considered as force transducers according to ISO376 in the range above the traceable force scale of PTB. Force transducers along with an indicator provide a 'deflection' $X$ but not a 'correct force' $F$.

For these reasons a consistent linguistic usage is needed for the investigation of BU-systems, which describes the signals and the derived, derivative quantities independent of their usage.

Tab.1: Designation of BU-system - quantities

\begin{tabular}{|c|c|}
\hline $\begin{array}{l}\text { Symbol / Unit } \\
\text { [source] }\end{array}$ & (short) english designation \\
\hline$F_{\mathrm{R}} / \mathrm{N}$ & real acting force \\
\hline $\begin{array}{l}F_{\mathrm{i}} / \mathrm{N} \\
\text { [DIN EN ISO } \\
\text { 7500] }\end{array}$ & indicated machine force \\
\hline$F_{\mathrm{LS}} / \mathrm{N}$ & load step \\
\hline$F_{I S} / \mathrm{N}$ & indicated single transducer force \\
\hline$F_{\mathrm{IB}} / \mathrm{N}$ & indicated build-up system force \\
\hline$F_{\mathrm{S}} / \mathrm{N}$ & sum force \\
\hline$F_{\mathrm{RS}} / \mathrm{N}$ & reference sum force \\
\hline$d s$ & $\begin{array}{l}\text { deviation of BU-system sum } \\
\text { force }\end{array}$ \\
\hline$d \mathrm{LS}$ & $\begin{array}{l}\text { relative deviation of load and } \\
\text { sum force }\end{array}$ \\
\hline$d_{\mathrm{LI}}$ & $\begin{array}{l}\text { relative deviation of indicated } \\
\text { BU-system force }\end{array}$ \\
\hline$d_{\mathrm{k}}$ & $\begin{array}{l}\text { deviation by parallel connection } \\
\text { of force transducers }\end{array}$ \\
\hline & (connection deviation) \\
\hline
\end{tabular}

\section{Generating bu-system measurement data}

To answer the question whether or not the behavior of a bu-system can be computed only by the calibration results of the single transducers, a complex measurement and evaluation procedure has to be performed. Fig. 2 shows this procedure as well as the resulting quantities. The diagram is completed by the information given in tab. 1. Finally the data has to be processed to be comparable since there are results from different machines at different particular loads.

A BU-system consists of at least three force transducers. The single calibration results of these force transducers form the basis for evaluation of the overall system. These allow the calculation of a force by a compensation polynomial according to ISO376, which will be hereinafter referred to as indicated single transducer force, FIS.

For a complete evaluation of a BU-system four parameters are defined, each representing a force.

As a reference the indicated machine force is used. In contrast, the indicated force of the BUsystem is provided, which can be determined in two different ways.

One way is to calibrate the system so that a single signal is generated. This happens either by the use of a sum box (an electrical component comprising the arithmetic mean of the input signals at the output by a suitable interconnection of these) or by arithmetic averaging of the signals from the single transducers. This signal can be evaluated according to ISO376 which results in a polynomial that enables the calculation of a force based on the mean signal of the BU-system. For this force "indicated BU-system force" is agreed, because this force value is indicated by a commercial BU-system after calibration using a sum box. The indicated BU-system force is considered as the force value primarily displayed by a BU-system.

The second way is to use the already existing calibration results of the single transducers to determine three individual force signals, which then are summed. This way, for example, is appropriate when the BU-system is not calibrated in the rated load range because its nominal load exceeds the traceability of the force. For this force, the term "BU-system sum force" is used because this value is mathematically determined by summation.

When force transducers are calibrated individually they experience fundamentally different boundary conditions of force compared to a calibration of the entire system. To quantify this difference, it is necessary to establish another reference value. For this quantity, the term "reference sum force" is agreed. It applies as the sum of the indicated single transducer forces gained during independent single calibration.

\section{Characteristics of BU-system}

After computing the four force values that are provided by a BU-system or its components, the deviations between them are to be calculated. This applies as follows.

For the deviation between the indicated BUsystem force and the BU-system sum force, the 


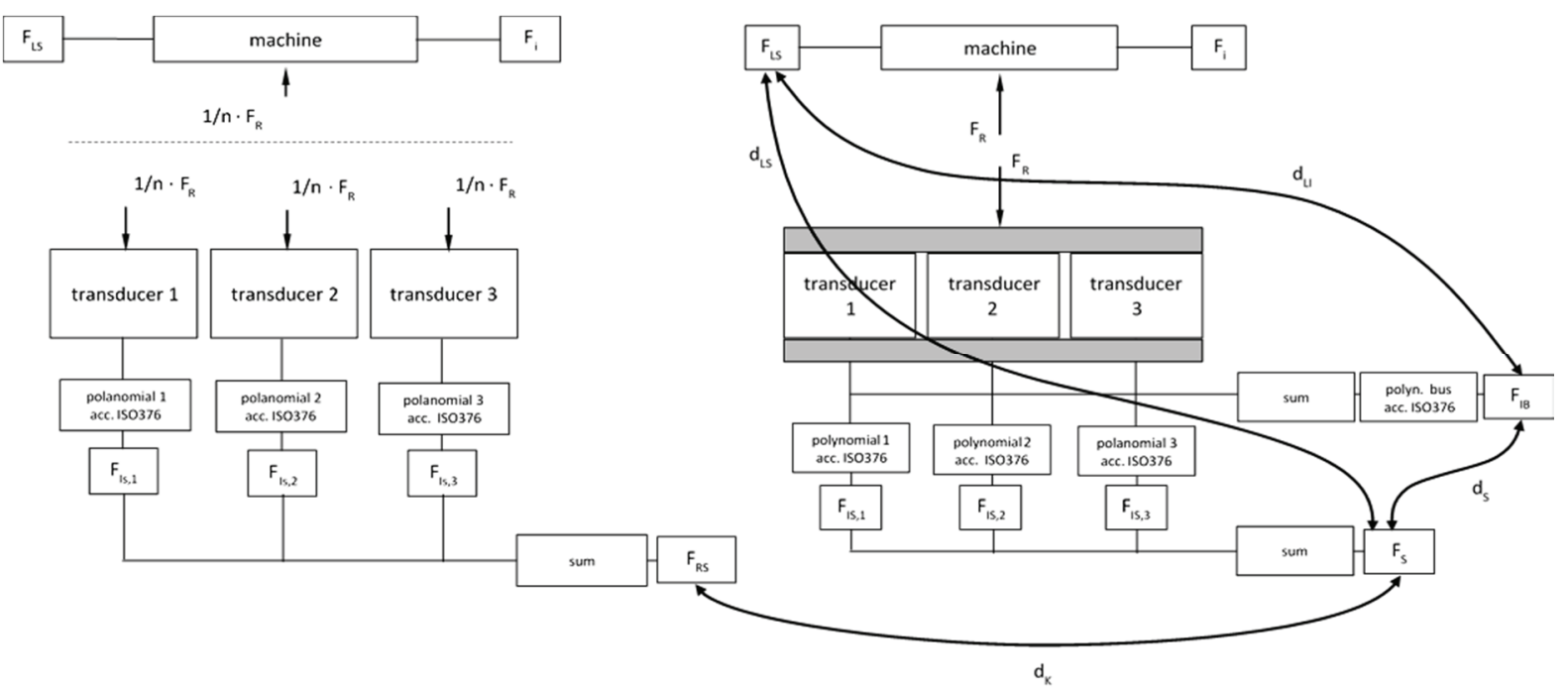

Fig. 1. scheme of the evaluation of bu-system investigation, showing all relevant quantities and their correlation

term "relative deviation of BU-system sum force" or shortened within an appropriate context "sum deviation" is agreed, what applies

$$
\begin{aligned}
& d_{\mathrm{S}}=\frac{F_{\mathrm{IB}}-F_{\mathrm{S}}}{F_{\mathrm{S}}} \cdot 100 \\
& d_{\mathrm{S}}=\left(d_{L I}-d_{L S}\right) \frac{F_{L S}}{F_{S}} \cdot 100
\end{aligned}
$$

to be indicated in percent.

For the deviation between the sum force of the $\mathrm{BU}$-system and the load step, the term "relative deviation of load and sum force" is agreed or shortened within an appropriate context "loadsum deviation" for which the following applies,

$$
d_{\mathrm{LS}}=\frac{F_{\mathrm{S}}-F_{\mathrm{LS}}}{F_{\mathrm{LS}}} \cdot 100
$$

For the deviation of the indicated BU-system force to the load step, the term "relative deviation of indicated BU-system force" is agreed, for which applies

$$
d_{\mathrm{LI}}=\frac{F_{\mathrm{IB}}-F_{\mathrm{LS}}}{F_{\mathrm{LS}}} \cdot 100
$$

The actual benchmark for assessing the deviation between the individual and sum measurement is the relative deviation of the reference sum force to the BU-system sum force. Since there is a systematic deviation that arises by combining force transducers to a BUsystem by parallel interconnection, for this quantity, the term "relative deviation by parallel combination of force transducers" is agreed or shortened within an appropriate context "combination deviation", for which applies

$$
d_{\mathrm{K}}=\frac{F_{\mathrm{RS}}-F_{\mathrm{S}}}{F_{\mathrm{S}}} \cdot 100
$$

\section{Processing of obtained data}

Since exactly given force values cannot be reached precisely when performing measurements in testing machines, which is due to their individual principle of generating forces, the actually achieved force values must be interpolated to rounded force values that are used for the respective comparison. However, the individual forces acting on the single transducer within a BU-system are unknown. Therefore, the load distribution, which is shown by the indicated single transducer force in BUsystem measurement is used for the computation of the distribution of the indicated machine force. This means that the indicated single transducer forces are calculated using their individual single calibration results and the indicated machine force is assumed as acting on the transducers in the same ratio.

After the load distribution on the individual load cell inside the BU-system is determined, there are now force values according to each measurement value. The force values are always different, however, and thus are not comparable.

Therefore, the force values must be interpolated to comparable, rounded values, the corresponding measured values are converted according to the same context.

For an ISO 376 evaluation of the mean signal of the BU-system it is advisable, to use the mean of the interpolated force values and transducer signals as described before. 


\section{Influence of design parameters of bu- systems}

Since any transducer has a more or less large sensitivity to cross forces and bending moments, the mechanical decoupling of these parasitic loads is an important issue. In fig. 2 the result of a numerical simulation of the cross force influence on a $10 \mathrm{MN}$ transducer is shown. The change of the signal $S$ is plotted against the cross force $Q$.

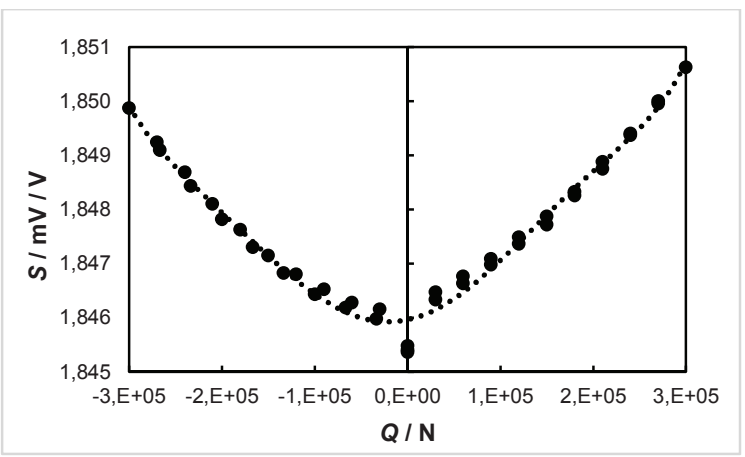

Fig. 2: numerical simulation of the influence of a cross force of $\pm 300 \mathrm{kN}$ on the transducer signal under maximum load of $10 \mathrm{MN}$

The cross force sensitivity of the transducer combined with particular construction layouts lead to an increasing load sum deviation of the bu-system.

High cross forces can be generated if the interconnection plates have a large radius. This leads to large relative lever lengths from the force introduction into the system to the force introduction into the transducer. A consequence is a high elastic deformation of the plates and by that a high generation of cross forces. Now, when a load cell is utilized, which has a high sensitivity to cross forces, there follows a strong deviation of the measurement results.
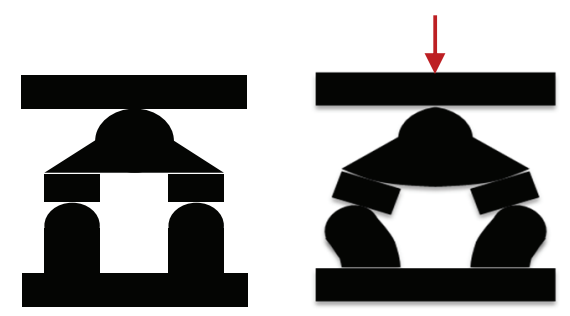

Fig. 3: principle of generating cross forces due to elastic behavior of interconnection plates

This principle is illustrated in fig. 3. For the design of bu-systems it has to be carefully weighed between the radius of the entire system which is bending by the diameter of the transducer and the cross force sensitivity of the transducer.
Usually bending ring transducers are considered as less sensitive against these parasitic loads compared to strain cylinders. In contrast strain cylinders can be designed with very small diameters compared to bending ring transducers, especially in the highest force range.

Tab.2: Designation of BU-system - quantities

\begin{tabular}{|c|cc|}
\hline $\begin{array}{c}\text { Load Step / } \\
\text { kN }\end{array}$ & low & high \\
\hline \hline 1500 & $0,131 \%$ & $-0,020 \%$ \\
3000 & $0,145 \%$ & $-0,005 \%$ \\
4500 & $0,150 \%$ & $0,004 \%$ \\
6000 & $0,151 \%$ & $0,010 \%$ \\
7500 & $0,150 \%$ & $0,011 \%$ \\
9000 & $0,149 \%$ & $0,014 \%$ \\
10500 & $0,147 \%$ & $0,012 \%$ \\
12000 & $0,145 \%$ & $0,013 \%$ \\
13500 & $0,145 \%$ & $0,013 \%$ \\
15000 & $0,144 \%$ & $0,014 \%$ \\
16500 & $0,140 \%$ & $0,011 \%$ \\
\hline
\end{tabular}

Tab. 2 shows the results of a measurement series using two different interconnection plates. As easily can be seen the relative deviation of load and sum force can be reduced by approximately a factor of ten.

\section{Conclusion}

The shown results lead to the following. Since the deviation between single transducer force signals and the bu-systems mean force signal is apparently caused largely by the kinematic behavior of the system, it is a systematic error. A systematic error can be computed and taken into account when estimating the bu-systems uncertainty. When investigating a bu-system concerning its uncertainty it is necessary to firstly investigate its elastic behavior carefully and to figure out what kind of parasitic forces are generated. The next step will be to measure the sensitivity of the utilized transducers in order to create a basis for the mathematical compensation of the load sum deviation. Therefore a special facility is needed.

\section{References}

[1] Dr. Rolf Kumme, Force traceability within the meganewton range, IMEKO International Conferences, February 2014

[2] DIN EN ISO 376:2011-9 Metallische Werkstoffe - Kalibrierung der Kraftmessgeräte für die Prüfung von Prüfmaschinen mit einachsiger Beanspruchung 
[3] DIN EN ISO 7500-1:2014-04 Metallische

Werkstoffe - Prüfung von einachsigen

Prüfmaschinen, Teil 1 